\title{
Article
}

\section{Orientation and location of the finite helical axis of the equine forelimb joints}

Kaashoek, Mariëlle, Hobbs, Sarah Jane, Clayton, Hilary Mary, Aerts, Peter and Nauwelaerts, Sandra

Available at http://clok.uclan.ac.uk/28029/

Kaashoek, Mariëlle, Hobbs, Sarah Jane ORCID: 0000-0002-1552-8647, Clayton, Hilary Mary, Aerts, Peter and Nauwelaerts, Sandra (2019) Orientation and location of the finite helical axis of the equine forelimb joints. Journal of Morphology, 280 (5). pp. 712-721. ISSN 0362-2525

It is advisable to refer to the publisher's version if you intend to cite from the work. http://dx.doi.org/10.1002/jmor.20978

For more information about UCLan's research in this area go to http://www.uclan.ac.uk/researchgroups/ and search for <name of research Group>.

For information about Research generally at UCLan please go to http://www.uclan.ac.uk/research/

All outputs in CLoK are protected by Intellectual Property Rights law, including Copyright law. Copyright, IPR and Moral Rights for the works on this site are retained by the individual authors and/or other copyright owners. Terms and conditions for use of this material are defined in the policies page. 
1 Orientation and location of the finite helical axis of the equine

2 forelimb joints

3

4 Helical axis of the equine forelimb joints

5

6 Mariëlle Kaashoek ${ }^{1}$, Sarah Jane Hobbs ${ }^{2}$, Hilary Mary Clayton ${ }^{3}$, Peter Aerts ${ }^{1,4}$ and Sandra

$7 \quad$ Nauwelaerts ${ }^{1,5}$

8

9 1) Functional Morphology lab, Biology Department, University of Antwerp, Campus Drie 10 Eiken, Building D, Belgium

2) Centre for Applied Sport and Exercise Sciences, University of Central Lancashire, Preston, Lancashire, United Kingdom

3) Department of Large Animal Clinical Sciences, Michigan State University, East Lansing,

MI, United States of America

4) Department of Movement and Sports Sciences, University of Ghent, Belgium

5) Centre for Research and Conservation Antwerp Zoo, Antwerp, Belgium

17

\section{Corresponding author:}

Mariëlle Kaashoek

Marielle.kaashoek@uantwerpen.be

Functional Morphology lab, Biology Department, University of Antwerp, Campus Drie 
Abstract

To reduce anatomically unrealistic limb postures in a virtual musculoskeletal model of a horse's forelimb, accurate knowledge on forelimb joint constraints is essential. The aim of this cadaver study is to report all orientation and position changes of the finite helical axes (FHA) as a function of joint angle for different equine forelimb joints. Five horse cadaver forelimbs with standardized cuts at the midlevel of each segment were used. Bone pins with reflective marker triads were drilled into the forelimb bones. Unless joint angles were anatomically coupled, each joint was manually moved independently in all three rotational DOFs (flexion-extension, abduction-adduction, internal-external rotation). The three-dimensional coordinates of the marker triads were recorded using a six infra-red camera system. The FHA and its orientational and positional properties were calculated and expressed against joint angle over the entire range of motion using a finite helical axis method. When coupled, joint angles and FHA were expressed in function of flexion-extension angle. Flexion-extension movement was substantial in all forelimb joints, the shoulder allowed additional considerable motion in all three rotational DOFs. The position of the FHA was constant in the fetlock and a constant orientation of the FHA was found in the shoulder. Orientation and position changes of the FHA over the entire ROM were observed in the elbow, carpus and PIP-DIP joints. We report FHA position and orientation changes as a function of flexion-extension angle to allow for inclusion in a musculoskeletal model of a horse to minimize calculation errors caused by incorrect location of the FHA. 
Keywords: Musculoskeletal Model, Range of Motion, Helical Axis,

\section{Research Highlights and Graphical Abstract}

When measuring the finite helical axes of the equine forelimb joints over their entire range of motion, changes in orientation were observed in the elbow, carpus, fetlock and PIP-DIP joints. Changes in position were measured in the shoulder, elbow, carpus and PIP-DIP joints.

Main Text

\section{Introduction}

A common way to study the locomotion of an animal is conducting in vivo gait experiments where external force and segment/joint movement patterns are typically reported. The underlying mechanisms to generate locomotion, such as muscle control, internal loading of anatomical structures, joint forces and muscle energetics are more difficult to obtain using non-invasive techniques (Umberger \& Caldwell, 2013). One solution is the use of musculoskeletal models, which are three-dimensional virtual reconstructions of the musculoskeletal system that can estimate these internal variables (Umberger \& Caldwell, 2013). Such models use a linked segment approach of rigid bodies (bones) that connect at joints, and may include muscles, ligaments and other structures (Delp \& Loan, 1995; Seth, Sherman, Reinbolt, \& Delp, 2011). 
The three-dimensional motion of two adjacent segments can be described by defining their finite helical axis (FHA) (SHIAVI et al 1987), which describes the motion of two segments as a rotation about and a translation along an axis (Blankevoort et al 1990). In the models, joint constraints like the FHA are used to eliminate kinematics which are outside the range of natural poses or movements (Kambic, Roberts, \& Gatesy, 2017).

Different methods can be used to determine the axis of rotation of a joint, for example the symmetrical axis of rotation approach, finite helical axis and instantaneous helical axis methods, the latter two are amongst the most commonly used methods (Ehrig \& Heller, 2019). The In current study, the finite helical axis (FHA) was used to calculate the joint axes, this method uses three-dimensional motion data of two adjacent segments to determine the position and orientation of the FHA (Ehrig \& Heller, 2019; Shiavi et al., 1987). A clear progression of the FHA in a joint can be obtained by dividing the entire ROM into multiple windows and calculating the FHA over each window (Blankevoort, Huiskes, \& de Lange, 1990; Spoor \& Veldpaus, 1980; van den Bogert, Reinschmidt, \& Lundberg, 2008). Throughout movement, the FHA can shift and/or change in orientation, for instance in the human knee, where the position of the FHA changes during flexion (Blankevoort et al., 1990; van den Bogert et al., 2008). Orientation changes of the FHA occur when a joint rotates about two or three of the orthogonal axes simultaneously. Translations of the FHA can be observed when one bone slides along one or more of the three orthogonal axes. 
91

92

An accurate location of the three-dimensional FHA is important when building musculoskeletal models (Bru \& Pasqui, 2010). The kinematic and kinetic output of the model are directly affects when the position and/or orientation of the modelled joint axis deviates from the actual joint axis (Holden \& Stanhope, 1998; Stagni, Leardini, Cappozzo, Benedetti, \& Cappello, 2000). Furthermore, errors will be caused in the calculation of moment arms and joint moments (Camomilla et al., 2017). So far, forelimb joints of the equine athlete have been modelled using one degree of freedom joint descriptions. Previous models of Brown et al. (2003) and Swanstrom et al. (2005) describe the translations of the joint axes in craniocaudal/dorsopalmar and proximodistal as a function of flexion-extension angle. In the current study, we expand on these data by measuring multiple horses and adding the orientation properties of the FHA over the entire ROM for all equine forelimb joints to the description of the craniocaudal/dorsopalmar and proximodistal position of the FHA using a helical axis approach. The three-dimensional data of the FHA are reported as a function of joint angle and can be used when constructing a musculoskeletal model of the horse's forelimb.

\section{Material and Methods}

\section{Subjects}

Five horse cadavers (age: $16.75 \pm 1.35$ years, weight $532 \pm 6.58 \mathrm{~kg}$, varying breeds) were obtained from the pathology department at Ghent University, where the experiments were performed between April 2016 and November 2018. A formal ethical approval was waived by the chairperson of the ethical committee, based on Belgian and European 
legislation (EU directive 2010/63/EU), as all tissues were derived post mortem from the necropsy room or from a commercial abattoir. The horses included in this study did not show any signs of musculoskeletal injuries and either died of natural causes or were euthanized for non-locomotor issues. The cadavers were stored in a cooler at 4 degrees Celsius and experiments were performed approximately three days post mortem.

\section{Preparation of Specimens}

Each left forelimb was removed from the trunk by cutting the soft tissues between the scapula and the rib cage. Six forelimb segments were defined, from proximal to distal, the shoulder, brachium, antebrachium, metacarpus, pastern and hoof (Fig 1). To ensure that all anatomically possible positions and orientations of the distal segment with respect to the proximal segment in each joint were obtained and to maximize the ROM, standardized cuts through the soft tissue were made midway along the length of each forelimb segment to eliminate muscle, tendon, fascia and skin stiffness (Fig 1). Joint capsules, tendon attachment sites and ligaments surrounding the joint were kept intact.

A bone-pin was drilled into the shaft of the main bone of each segment: scapula (shoulder), humerus (brachium), fused radius and ulna (radio-ulna) (antebrachium), third metacarpal bone (metacarpus), proximal phalanx (pastern) and distal phalanx via the hoof wall (PIP-DIP joints) (Fig 1). Reflective marker triads with a marker diameter of $15 \mathrm{~mm}$, were attached to the bone pins. The secure attachment of the marker triads ensured that they represented the exact movements of the bones to which they were attached. The joints were, from proximal to distal, the shoulder, elbow, carpus, fetlock 
and PIP-DIP joints, which included the proximal and distal interphalangeal joints (Fig 1).

The FHA of the distal sesamoid bones were not tracked in this study.

\section{Dynamic Trials}

The limbs were either placed horizontal on a table with the lateral side facing upwards or partly lifted from the table depending on the rotational degree of freedom (rDOF) (flexion-extension, abduction-adduction, internal-external rotation) that was measured. Dynamic trials were conducted where each joint was manually moved through its full ROM in each of the three rDOFs separately. Each dynamic trial consisted of at least three movement cycles in one of the three rDOFs for a specific joint. The abduction-adduction and internal-external rotation trials were performed at one position of the flexionextension angle, except for the carpus which was measured in two positions, at extension $\left(\sim-0^{\circ}\right)$ and at flexion $\left(\sim 90^{\circ}\right)$. During the manual movement of the joints the segments were moved until the experimenter was not able to move the limb any further in that direction due to bone-bone or muscle-skin contact. Out of plane motion was kept to a minimum while still allowing motion in the other rDOF in case two or more rDOF were coupled (Kambic et al., 2017). The three-dimensional positions of all markers were recorded within a pre-calibrated field of view $(\sim 1.5 \mathrm{~m} \times 1.5 \mathrm{~m} \times 1 \mathrm{~m}$, mean camera residual $\leq 0.35$ ) using a motion analysis system with six infra-red cameras recording at $60 \mathrm{~Hz}$ (VICON, Los Angeles, CA, USA) and Vicon Workstation software.

\section{Defining the local coordinate systems}


After the dynamic trials, soft tissues were removed to expose the bones. Bones were disarticulated, and four anatomical markers were placed at standardized locations on the joint surfaces (Fig 2): the first two markers were placed at the centre of the proximal and distal articular surfaces; the third and fourth markers were located in the middle of the lateral side and in the middle of the caudal/palmar side of the distal articular surface. A static recording was made that captured the position of the triad markers with respect to the anatomical markers.

An anatomically relevant, right-handed local coordinate system (LCS) was defined for each forelimb segment using the anatomical markers (Fig 2). The origin of the LCS was placed in the centre of the distal articular surface using the first anatomical marker (Grood \& Suntay, 1983). The proximodistal-axis ran through the long axis of the bone and was positive in the proximal direction. The craniocaudal/dorsopalmar-axis pointed from the origin towards the caudal side of the segment. The mediolateral-axis ran transversely and was positive toward the lateral side.

\section{Pre-Analysis}

The virtual marker coordinates from the dynamic trial and the static recording were tracked using the Vicon Workstation software. The coordinate data were filtered using a fourth-order Butterworth filter with a cut-off frequency of $5 \mathrm{~Hz}$. The orientation matrices and displacement vectors between the tracking markers of the static file and the dynamic trials were calculated using singular value decomposition (Söderkvist \& Wedin, 1993). Coordinates of the anatomical markers of both segments were 
transformed to the global coordinate system (GCS) of the dynamic trials. Calibration of the Vicon camera system was performed using a T-wand and an L-frame, the position of the latter was used to define the GCS. The LCS of the proximal (P-LCS) and distal (D-LCS) segments were determined using the transformed anatomical marker coordinates. For each frame, the P-LCS was translated to the origin of the GCS and rotated to align with the orientation of the axes of the GCS. The D-LCS was expressed relative to the P-LCS.

\section{Analysis: Joint angles and Range of Motion}

Kinematic analyses were performed using the MATLAB (The MathWorks Inc, Natick, Massachusetts) based software package KineMat (Reinschmidt \& van den Bogert, 1997), based on the work of Grood \& Suntay (1983). The orientations of the D-LCS with respect to the P-LCS were used to calculate the joint Cardan angles, which were defined by rotations that occurred about three axes: the first rotation was flexion-extension about the mediolateral-axis of the proximal segment, the second rotation was abductionadduction about the floating axis, which was the result of the cross product between the mediolateral-axis of the proximal segment and the proximodistal-axis of the distal segment. The third rotation was internal-external rotation about the proximodistal-axis of the distal segment (Grood \& Suntay, 1983; Zatsiorsky, 1997).

Data from the dynamic trials, for each type of rotation, were used to determine the minimal and maximal joint angle and the ROM for each rDOF. Joint angles were calculated using the neutral positions of the forelimb joints as reported in Weller et al 2006, (Fig. 1). During the quality control, trials were removed from the data set when 
for example bone pins appeared to be loose, misplacement of the anatomical markers led to untenable joint angles or when the out of plane motion showed large deviations when testing for a certain rDOF.

\section{Analysis: Helical axis}

Data from the dynamic trials and the corresponding static recordings were used to calculate the FHA. From each trial, one movement cycle was selected. The increasing and decreasing joint angle phases of the movement cycle were divided into equal steps of approximately 5 degrees. Using the KineMat software package (Reinschmidt \& van den Bogert, 1997), based on Spoor \& Veldpaus (1980), FHA's were calculated for each step (van den Bogert et al., 2008). A homogeneous transformation matrix (T) was calculated between the transformed D-LCS of $\theta$ and $\theta \pm \sim 5$ degrees using singular value decomposition. Properties of the FHA were extracted from T using the method of Spoor and Veldpaus (1980) adapted by Reinschmidt and van den Bogert (1997).

Depending on the rDOF that was measured, the position of the FHA was calculated as the distance between the origin and the intersection of the FHA with the plane perpendicular to the FHA which was then projected on the associated P-LCS axis (Fig 3). The planes used for the different trials were the sagittal plane for FE trials, the frontal plane for AA trials and the transverse plane for IE trials (Fig 3A-B). The proximodistal position of the FHA was the distance between the intersection of the FHA with associated plane projected onto the proximodistal axis and the origin of the P-LCS (Fig $3 A B)$. The distance between the intersection of the FHA with the associated plane 
projected onto the craniocaudal/dorsopalmar axis and the origin of the P-LCS (Fig 3BC).

The medio-lateral distance was defined as the distance between the intersection of the FHA and the associated plane projected onto the mediolateral axis and the origin of the P-LCS (Fig 3AC).

The deviation angle of the FHA was the angle between the projection of the FHA onto the transverse plane of the proximal segment and the mediolateral axis of the P-LCS (Fig 3D). The angle between the FHA projected onto the sagittal plane of the proximal segment and the proximodistal axis was defined as the inclination angle (Fig 3E) (Blankevoort et al., 1990).

\section{Statistical analysis}

The five variables describing the position and orientation of the FHA (inclination, deviation, craniocaudal/dorsopalmar position, proximodistal position and mediolateral position (Fig 3)) were analysed statistically using SPSS (SPSS version 24.0; SPSS Inc, Chicago, Illinois). A multivariate analysis of covariance (MANCOVA) test was performed on the set of five dependent variables, inclination angle, deviation angle, craniocaudal/dorsopalmar position, proximodistal position and mediolateral position for each joint with joint angle of the tested rDOF as a covariate and leg number (individual) and direction of movement as fixed, independent factors. The latter was added to test whether the FHA variables were influenced by the direction of movement. 
To study the effect of joint angle on each of the FHA variables for each joint for the horse as a species, leg number was removed from the MANCOVA and either a regression equation or a mean value was calculated over all limbs for the FHA variable depending on the outcome of the MANCOVA, Table 4-5. Mean values for FHA variables were calculated over the entire ROM over all limbs when there only was a significant effect of leg number (Table 4). If there was a significant effect of joint angle or for the interaction effect between leg number and joint angle, i.e. the FHA changed with joint angle, subsequent reduced major axis regression analyses were performed using JMP (JMP ${ }^{\circledR}$, Version 13.2.1 SAS Institute Inc., Cary, NC, 1989-2019) to determine the amount of change of the variable over the entire ROM (Table 4-5).

The interaction between rDOF can be a complex relationship in a three-dimensional space, however for this study we only calculated the individual relationships between the rDOF (Kambic et al., 2017). Pearson correlation tests were performed on all three rDOFs for each trial to test whether there was a coupling between the rDOFs, Table 6 . An reduced major axis regression was calculated if there was a significant correlation between two rDOFs.

\section{Method validation}

Prior to conducting the experiments, the analysis script developed for this study and experimental setup were validated using an artificial test joint with one rDOF (i.e. a door hinge connecting two wooden segments). The location of the FHA should be stable and 
running through the centre of the door hinge, indicated with extra markers, for the experiment and analysis to be correct.

\section{Results}

\section{Joint angles and range of motion}

The results for the joint angles and ROM and the out of plane motion for each of the forelimb joints are reported in Table 1-3. The variation in the ROM of the carpus, fetlock and PIP-DIP joints were smaller compared to the variation of the shoulder and elbow. In the distal joints, the endpoints were determined by bone to bone contact and in the proximal joints the endpoints were influenced by the amount of muscle tissue surrounding the joints. The ROM values reported in this study were aimed to be larger than those reported in kinematic studies and exceeded the normal physiologic ranges (Back et al., 1995).

\section{Finite helical axis}

The FHA for the artificial joint (i.e. a door hinge connecting two wooden segments) was located, as expected, at the same location as the door hinge and did not show any change in orientation or translation when changing the angle between the wooden segments.

The FHA was calculated for all rDOFs with a ROM above 25 degrees in order to obtain a clear progression of the FHA over the entire ROM. Results of the statistical tests regarding leg number and joint angle are reported in Table 4-5. Locations of the FHA 
were not calculated for the axis about which the rotation occurred. A significant effect of the direction of movement was only found for the deviation angle when moving the shoulder in flexion and extension $\left(P_{\cup D^{*} J A}=0.02\right)$, the inclination angle for the internal external rotation trials for the shoulder $\left(\mathrm{PUD}^{*} \mathrm{JA}_{\mathrm{A}}=0.02\right)$, proximodistal position in the flexion-extension trial of the carpus $\left(P_{U D^{*} J A}=0.00, P_{U D}=0.00\right)$ and for the inclination angle while moving the fetlock in flexion-extension $\left(P_{U D^{*} J A}=0.03\right)$ and for the dorsopalmar position while moving the PIP-DIP in flexion-extension ( $\left.\mathrm{PUD}^{*} \mathrm{JA}=0.03\right)$.

Results of the orientation (inclination and deviation) and positional (craniocaudal/dorsopalmar, proximodistal and mediolateral position) of the FHA over the entire ROM are shown in Table 4. The position and orientation of the FHA remained constant over the entire ROM when moving the shoulder through abduction-adduction and internal-external rotation. FHA position and orientation reported below were determined by calculating the difference between minimal and maximal joint angle using the regression equations reported in Table 5. The FHA of the elbow and fetlock showed a significant change in inclination (elbow $=46^{\circ}$, fetlock(up) $=71^{\circ}$ fetlock (down) $\left.=56^{\circ}\right)$ and deviation angle $\left(\right.$ fetlock $\left.=6^{\circ}\right)$, whereas the FHA position was constant for both joints. When moving the shoulder in flexion-extension, only the orientation changed significantly. Changes in proximodistal ( $u p=8 \mathrm{~mm}$, down $=26 \mathrm{~mm})$ and dorsopalmar $(40$ $\mathrm{mm}$ ) position were found for the flexion-extension trials of the carpus, which also showed change in inclination $\left(-60^{\circ}\right)$ and deviation $\left(9^{\circ}\right)$ angle. The PIP-DIP joints displayed a change in inclination angle $\left(-144^{\circ}\right)$ and in proximodistal position (up $=3 \mathrm{~mm}$, down $=$ $8 \mathrm{~mm})$ 
No significant correlations were found between flexion-extension and internal-external rotation in the shoulder, carpus and PIP-DIP joints and internal-external rotation and flexion-extension for the shoulder, Table 6. Weak correlation (significant correlation with a Pearsons correlation between 0.3-0.65) were found between both rDOF when moving the shoulder in abduction-adduction and between flexion-extension and abduction-adduction for the shoulder, elbow, carpus, fetlock and PIP-DIP joints, Table 6. The fetlock also showed weak correlations between flexion-extension and internalexternal rotation. Internal-external rotation and abduction-adduction for the shoulder and flexion-extension and internal-external rotation for the elbow showed a strong correlation (significant correlation with a Pearsons correlation above 0.65). Reduced major axis regression equations describing the correlation are reported in Table 6 which can be used when modelling the forelimb joints in musculoskeletal models.

\section{Discussion}

In the current study, we describe the three-dimensional properties of the FHA over the entire ROM for all forelimb joints as a function of joint angle. As expected, the shoulder displayed substantial rotation in abduction-adduction and internal-external rotation due to its joint surface morphology. For abduction-adduction the FHA of the shoulder showed no orientational or positional changes, orientational changes were observed when moving the shoulder in flexion-extension, orientational and position changes were found for internal-external rotation. The FHA of the elbow and fetlock only showed orientation changes. The carpus and PIP-DIP joint displayed both orientation and 
position changes. Most of the joints also showed significant correlations between the rDOFs except between flexion-extension and internal-external rotation for the shoulder.

Equine forelimb joints moved mainly in the parasagittal plane: only the shoulder allowed substantial extra-sagittal motion. The shoulder is classified as an ellipsoidal ball and socket joint (Budras, Sack, Röck, Horowitz, \& Berg, 2012). Due to the elongated elliptic shape of the glenoid cavity, the FHA translated significantly along the proximodistal axis when moving the joint through flexion-extension. Translations of the FHA were not found for the abduction-adduction trials. Previous models generally excluded the shoulder (Brown, Pandy, Kawcak, \& Mcilwraith, 2003; Michael D. Swanstrom, Zarucco, Hubbard, Stover, \& Hawkins, 2005), or only studied the motion of the shoulder within the sagittal plane making it difficult to compare all our findings. Leach et al. (1988) did found translations of the instant centre of rotation as a function of flexion-extension angle, however these were in the craniocaudal direction.

In contrast with the shoulder, movements of the elbow are mainly restricted to the parasagittal plane and are reflected in the morphology of their articular surfaces. In the elbow, a groove running along the centre of the articular surface fits into a matching ridge on the adjacent joint surface. These interlocking structures in combination with collateral ligaments restrict the joints to parasagittal plane motion only (Ross \& Dyson, 2011). The fetlock has a similar interlocking structure, however due to the shape of the condyle of the fetlock, which is larger on the medial side compared to the lateral side, there is more out-of-sagittal plane motion allowed compared to the elbow, which is also 
shown in our results (Table 1-3). Both showed changes in inclination angle, the fetlock also displayed a change in deviation angle when flexing the joint.

In the PIP-DIP joints, a saddle-like joint articulation morphology is found, allowing more out of sagittal plane motion compared to the interlocking structures of the elbow (Budras et al., 2012). This is also reflected in the larger out of sagittal plane ROM values of the PIP-DIP joints compared to the elbow (Table1-3). When comparing our results with three-dimensional kinematic studies, we found that the ROM values for the out of sagittal plane motion for the PIP-DIP joints was larger than observed in in vivo gait experiments (H. Chateau, Degueurce, \& Denoix, 2006; Henry Chateau, Degueurce, \& Denoix, 2004; Clayton, Sha, Stick, \& Robinson, 2007; Panagiotopoulou, Rankin, Gatesy, \& Hutchinson, 2016; Roach et al., 2014). However, these gait experiments were performed on a relative flat surface and at relative low locomotion speeds. Even though larger out of sagittal plane ROMs were measured, the FHA PIP-DIP joints did not show a significant change in deviation angle and the proximodistal position.

The PIP-DIP joints, the proximal and distal interphalangeal joint, were measured simultaneously because most gait analysis consider the first and second phalanx are a single segment (Back et al., 1995; Khumsap, Clayton, Lanovaz, \& Bouchey, 2002) and the musculoskeletal models for which the FHA results are reported will be driven by kinematic data. Previous detailed studies on the distal joints showed that there is a relative small amount of motion occurring at the proximal interphalangeal joint (Henry Chateau et al., 2004; Clayton et al., 2007) and translations of the distal interphalangeal 
joint were $\sim 1 \mathrm{~mm}$ (Michael David Swanstrom, 1998), for models that require such detailed data on the individual joints, we suggest undertaking an three-dimensional Xray study or using prior XROMM data from which the FHA can be determined in more detail for both joints individually (Panagiotopoulou et al., 2016; Roach et al., 2014). The same is suggested for the individual carpal bones, due to the complex movements of the individual carpal bones (Yalden, 1971).

The carpus was measured as one entity although it technically also consists of multiple joints. The radio-ulna is connected to the metacarpus III via two rows of carpal bones resulting in three joints, from proximal to distal, the antebrachiocarpal, middle carpal and carpometacarpal joints (Budras et al., 2012). From flexion to extension, the FHA translates simultaneously in both a distal and a dorsal direction which could be caused by the conformation of the articular surfaces of the distal end of the radius and the proximal rows of carpal bones and by the increased separation of the two proximal carpal joints on their dorsal side in flexion. Studies have shown limited movement at the carpometacarpal joint and the distal row of carpal bones has been attached to the proximal metacarpus in previous musculoskeletal models (Brown et al. 2003; Swanstrom et al. 2005).

Significant inter-limb variation was found in all forelimb joints, which most likely is partially due to the manual placement of the anatomical markers, which determines the position of the LCS. Small differences in the orientation of the LCS will lead to over or under estimation of the joint angles (Clayton, Sha, Stick, \& Mullineaux, 2004) but can 
410

411

also lead to variation in the position and orientation of the FHA. To obtain an accurate model of a specific horse, ideally the horse's own FHA data should be used. However, when building a generic horse model, this inter-limb variation is relatively small and can be neglected, the regression equations mentioned in Table 4 can be used to define the FHA in equine musculoskeletal models.

When comparing our results to previous reported instant centre of rotations, similar locations were found for the elbow and fetlock (Leach \& Dyson, 1988). Leach et al. 1988 also found a dorsopalmar displacement of the instant centre of rotation for the carpus. Comparing our results to previously reported data of musculoskeletal models proved difficult. Brown et al. (2003) does not provide enough detail on the locations of the coordinate systems to directly compare the location and focuses more on the muscle geometry. Some of our data contrasts with Swanstrom M.D (1998) probably caused by the differences in the number of subjects and the approach: we measured the carpus as one entity and Swanstrom separated the carpus in two parts (Leach \& Dyson, 1988). They also reported small translations in the fetlock which we were not able to detect. These small translations possibly disappeared in our dataset of five horses, whereas the Swanstrom (1998) had data for one horse, which did not allow for a statistical analysis. Differences were possibly also due to the use of different methods, MRI, CT and radiographs, versus bone pins. We also used a different calculation method, the helical axis method. A direct comparison of the absolute values of the centre of rotation was not possible because we used such different methods. 
In conclusion, in this study, we report the three-dimensional behaviour of the FHA, relative to the proximal segment of the different forelimb joints, as a function of the flexion-extension angle. The findings of this study should be taken into account when constructing a musculoskeletal model for an equine forelimb, however differences in the definition of the local coordinate systems between the model and this study should be taken into account.

\section{Author contributions}

Sarah Jane Hobbs and Hilary Mary Clayton conceived the original idea for this study and the methodology, they also proofread the manuscript prior to submission. Sarah Jane Hobbs also contributed to part of the analysis. Peter Aerts and Sandra Nauwelaerts supervised the project and were involved in proofreading the manuscript. Sandra Nauwelaerts also helped with the experiments, parts of the analysis and helped with the writing. Mariëlle Kaashoek carried out the experiments, performed the analysis and wrote the manuscript.

\section{Acknowledgements}

We would like to thank Koen Chiers, Leen Van Brantegem, Michiel Moors and Hélène Claeys from the pathology department at Ghent University for their kind help during the experiments. Big thanks to Jan Scholliers, Kristiaan D'Août and the FunMorphers that helped us. We would like to thank the owners for making this study possible. And finally, we would like to thank the Bijzondere Onderzoeksfonds (BOF DOC PRO ID-31518) and the University of Antwerp for funding this project. 


\section{References}

Back, W., Schamhardt, H. C., Savelberg, H. H. C. M., Van den Bogert, A. J., Bruin, G., Hartman, W., \& Barenveld, A. (1995). How the horse moves: 1. Significance of graphical representations of equine forelimb kinematics. Equine Veterinary Journal, 27, 31-38.

Blankevoort, L., Huiskes, R., \& de Lange, A. (1990). Helical axes of passive knee joint motions. Journal of Biomechanics, 23, 1219-1229.

Brown, N. A. T., Pandy, M. G., Kawcak, C. E., \& Mcilwraith, W. C. (2003). Force- and moment-generating capacities of muscles in the distal forelimb of the horse. Journal of Anatomy, 101-113.

Bru, B., \& Pasqui, V. (2010). Localisation of the Instantaneous Axis of Rotation in Human Joints. In Advances in Robot Kinematics: Motion in Man and Machine (pp. 195-196).

Budras, K.-D., Sack, W. O., Röck, S., Horowitz, A., \& Berg, R. (2012). Chapter 2: Thoracic Limb. In Anatomy of the Horse (pp. 4-15). London: Schluetersche.

Camomilla, V., Cereatti, A., Cutti, A. G., Fantozzi, S., Stagni, R., \& Vannozzi, G. (2017). Methodological factors affecting joint moments estimation in clinical gait analysis: A systematic review. BioMedical Engineering Online, 16, 1-27.

Chateau, H., Degueurce, C., \& Denoix, J. M. (2004). Evaluation of three-dimensional kinematics of the distal portion of the forelimb in horses walking in a straight line. American Journal of Veterinary Research, 65, 447-455.

Chateau, H., Degueurce, C., \& Denoix, J. M. (2006). Effects of egg-bar shoes on the 3dimensional kinematics of the distal forelimb in horses walking on a sand track. Equine Veterinary Journal, 38, 377-382.

Clayton, H. M., Sha, D. H., Stick, J. A., \& Robinson, P. (2007). 3D kinematics of the interphalangeal joints in the forelimb of walking and trotting horses. Veterinary and Comparative Orthopaedics and Traumatology, 20, 1-7.

Clayton, H. M., Sha, D., Stick, J. a, \& Mullineaux, D. R. (2004). Three-dimensional carpal kinematics of trotting horses. Equine Veterinary Journal, 36, 671-676.

Delp, S. L., \& Loan, J. P. (1995). A graphics-based software system to develop and analyze models of musculoskeletal structures. Computers in Biology and Medicine, 25, 21-34.

Ehrig, R. M., \& Heller, M. O. (2019). On intrinsic equivalences of the finite helical axis , the instantaneous helical axis, and the SARA approach . A mathematical perspective. Journal of Biomechanics, 84, 4-10.

Grood, E. S., \& Suntay, W. J. (1983). A Joint Coordinate System for the Clinical Description of Three-Dimensional Motions: Application to the Knee. Journal of Biomechanical Engineering, 105, 136.

Holden, J. P., \& Stanhope, S. J. (1998). The effect of variation in knee center location estimates on net knee joint moments. Gait Posture, 7, 1-6.

Kambic, R. E., Roberts, T. J., \& Gatesy, S. M. (2017). 3-D range of motion envelopes reveal interacting degrees of freedom in avian hind limb joints. Journal of Anatomy, 231, 906-920. 
Khumsap, S., Clayton, H. M., Lanovaz, J. L., \& Bouchey, M. (2002). Effect of walking velocity on forelimb kinematics and kinetics. Equine Veterinary Journal. Supplement, 34, 325-329.

Leach, D. H., \& Dyson, S. J. (1988). Instant centres of rotation of equine limb joints and their relationship to standard skin marker locations. Equine Veterinary Journal., Supplement, 113-9.

Panagiotopoulou, O., Rankin, J. W., Gatesy, S. M., \& Hutchinson, J. R. (2016). A preliminary case study of the effect of shoe-wearing on the biomechanics of a horse' s foot. PeerJ, 4, 1-25.

Reinschmidt, C., \& van den Bogert, A. J. (1997). KineMat: A MATLAB toolbox for the reconstruction of spatial marker positions and for the analysis of threedimensional joint movements. International Society of Biomechanics, /http://www.isbweb.org/software/movanal/kinemat/.

Roach, J. M., Pfau, T., Bryars, J., Unt, V., Channon, S. B., \& Weller, R. (2014). Sagittal distal limb kinematics inside the hoof capsule captured using high-speed fluoroscopy in walking and trotting horses. Veterinary Journal, 202, 94-98.

Ross, M. W., \& Dyson, S. J. (2011). Chapter 26: The biomechanics of the equine limb and its effect on lameness. In Diagnosis and Management of Lameness in the Horse, 2nd Edition (Revised, pp. 270-281). London: Elsevier Health Sciences.

Seth, A., Sherman, M., Reinbolt, J. A., \& Delp, S. L. (2011). OpenSim: A musculoskeletal modeling and simulation framework for in silico investigations and exchange. Procedia IUTAM, 2, 212-232.

Shiavi, R., Limbird, T., Frazer, M., Stivers, K., Strauss, A., \& Abramovitz, J. (1987). Helical motion analyses of the knee - I. Methodology for studying kinematics during locomotion. Journal of Biomechanics, 20, 459-469.

Söderkvist, I., \& Wedin, P.-Å. (1993). Determining the movements of the skeleton using well-configured markers. Journal of Biomechanics, 26, 1473-1477.

Spoor, C. W., \& Veldpaus, F. E. (1980). Rigid body motion calculated from spatial coordinates of markers. Journal Biomechanics, 13, 391-393.

Stagni, R., Leardini, A., Cappozzo, A., Benedetti, M. G., \& Cappello, A. (2000). E ! ects of hip joint centre mislocation on gait analysis results. Journal of Biomechanics, 33, 1479-1487.

Swanstrom, M. D. (1998). Joint Kinematics and Inertial Properties of the Thoroughbred Forelimb, M.S. thesis, University of California, Davis, CA.

Swanstrom, M. D., Zarucco, L., Hubbard, M., Stover, S. M., \& Hawkins, D. A. (2005). Musculoskeletal Modeling and Dynamic Simulation of the Thoroughbred Equine Forelimb During Stance Phase of the Gallop. Journal of Biomechanical Engineering, 127, 318.

Umberger, B. R., \& Caldwell, G. E. (2013). Musculoskeletal modeling. In Research methods in biomechanics, second edition (pp. 247-250). Champaign: Human Kinetics Publishers.

van den Bogert, A. J., Reinschmidt, C., \& Lundberg, A. (2008). Helical axes of skeletal knee joint motion during running. Journal of Biomechanics, 41, 1632-1638.

Weller, R., Pfau, T., May, S. A., \& Wilson, A. M. (2006). Variation in conformation in a cohort of National Hunt racehorses. Equine Veterinary Journal, 38, 616-621. 
545

546

547

548

549

550
Yalden, D. W. (1971). The functional morphology of the carpus in ungulate mammals. Actaanat, 78, 461-487.

Zatsiorsky, V. M. (1997). 2.1.5. Joint Rotation Convention. In Kinematics of Human Motion (pp. 98-101). 
551

552

553

554

555

556

557

558

559

560

561

562

563

564

565

566

567

568

569

570

571

572

Figure Legends

Fig 1. Schematic overview of the left equine forelimb. A) Schematic overview of the forelimb bones. B) Schematic overview of the experimental cadaver limbs. Segment boundaries are indicated with dark grey lines. Dashed red lines indicate the standardised locations of the mid-level cuts through the soft tissue. Bone pins were placed in the different forelimb bones as indicated in the figure. C) Overview of the joint angles at neutral position as reported in Weller et al. 2006. Grey circles indicate the extension angles and white circles indicate flexion angles of the joint.

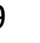

0

1

2

3

Fig 2. Schematic overview of the standardized anatomical marker locations, A) shows the marker locations in more detail on the dorsal joint surface and B) the overall marker placement. Grey spheres indicate the locations of the anatomical markers, marker numbers are shown inside the grey spheres. The origin of the bone is defined by the position of anatomical marker 1 . The proximodistal-axis, represented by the blue arrow, is positive in a proximal direction. The green arrow represents the mediolateral-axis which is positive towards the lateral side and the red arrow indicates the craniocaudal/dorsopalmar-axis which is positive in a caudal/palmar direction. 
574

575

576

577

578

579

580

581

582

Fig 3. Overview of the four FHA properties. The distance is defined as the distance between the intersection of the FHA (black dot) with the plane perpendicular to the FHA (grey) projected onto the proximodistal axis (blue) relative to the origin of the proximal segment (grey sphere). A) For FE, FHA intersection (black dot) with the sagittal plane (grey) projected onto the proximodistal axis (blue) for proximodistal distance and projected onto the mediolateral axis (green) for the mediolateral distance. B) For AA, FHA intersection (black dot) with the frontal plane (grey) projected onto the proximodistal axis (blue) for proximodistal distance and projected onto the cranial-caudal/dorsopalmar axis (red) for the cranial-caudal/dorsopalmar distance. C) For IE, FHA intersection (black dot) with the transverse plane (grey) projected onto the mediolateral axis (green) for mediolateral distance and projected onto the cranialcaudal/dorsopalmar axis (red) for the cranial-caudal/dorsopalmar distance. D) Deviation angle, the angle between the projection of the FHA (dashed line) onto the transverse plane (grey) of the proximal segment and the mediolateral-axis of the proximal segment (green arrow). E) Inclination angle, the angle between the projection of the FHA (dashed line) onto the sagittal plane of the proximal segment and the proximodistal-axis (grey) of the proximal segment. 